\title{
A importância do planejamento multidiscipilinar para correção do sorriso gengival: Relato de caso clínico.
}

\section{The Importance of Planning Multidisciplinary to Correct the Gummy Smile: A Case Report.}

\author{
Gabriela JoRge FARIA \\ Especialista em periodontia pela Pontifícia Universidade Católica de Minas Gerais, Belo Horizonte, MG, Brasil.
}

SÂMILA GonÇALVES BARRA*

Especialista em radiologia e imaginologia pela Universidade federal do Rio de Janeiro, Rio de Janeiro, RJ, Brasil.

Thais Ribeiral VIeIRA Mestre em clínicas odontológicas pela Pontifícia Universidade Católica de Minas Gerais, Belo Horizonte, MG, Brasil.

Peterson A. Dutra de Oliveira

Doutor em implantodontia pelo Centro de Ensino e Pesquisa São Leopoldo Mandic e professor adjunto IV da disciplina peridontia da Pontifícia Universidade Católica de Minas Gerais.

\begin{abstract}
RESUMO
Introdução: O sorriso gengival é definido pela exposição excessiva de gengiva maxilar durante o sorriso. A etiologia do sorriso gengival é variada e sua correta identificação é fundamental para confecção de um plano de tratamento eficaz. Para podermos diagnosticar precisamente o fator causador preponderante em cada caso, alguns aspectos clínicos devem ser avaliados. São esses: saúde periodontal, exposição dentária durante repouso, análise dimensional e funcional do lábio superior, mensuração da coroa clínica dos incisivos e caninos e harmonia entre os planos oclusais anteriores e posteriores. Além destas causas é de grande importância ressaltar a procura de tratamento pelo aspecto estético. Objetivo: Este artigo tem como objetivo apresentar um caso clínico destacando a importância do planejamento e da correção do sorriso gengival. Relato de caso: Paciente apresentou-se com queixa de "dentes curtos". Após os exames, o tratamento de escolha foi o aumento de coroa clinica no sextante anterior. Foi realizada gengivectomia, osteotomia e osteoplastia. Considerações finais: Novas alternativas menos invasivas têm sido encontradas com objetivo de se obter resultados semelhantes aos apresentados pelos tratamentos tradicionais, ou ainda melhores, promovendo, no entanto, um menor desconforto para o paciente. Assim, um planejamento e diagnóstico adequados são essenciais para que o melhor tratamento possa ser oferecido aos pacientes.

Palavras Chaves: Gengiva, Gengivoplastia, Osteotomia, Periodonto, Sorriso.
\end{abstract}

\begin{abstract}
Introduction: gummy smile is defined by excessive exposure of gum jaw during smile. The etiology of the gummy smile is varied and the correct identification is critical to making an effective treatment plan. In order to precisely diagnose the major causative factor in each case, some clinical aspects must be evaluated. These are: periodontal health, dental exhibition during rest, dimensional and functional analysis of the upper lip, measurement of clinical crown of incisors and canines and harmony between the anterior and posterior occlusal planes. Apart from these causes is very important to emphasize seeking treatment for aesthetics. Objective: This article aims to present a case highlighting the importance of planning and the gummy smile correction. Case report: Patient presented with complaint of "short teeth." After the tests, the treatment of choice was the increase of clinical crown in the previous sextant. It was carried out gingivectomy, osteotomy and osteoplasty. Concluding Remarks: New less invasive alternative has been found in order to obtain results similar to those presented by the traditional treatments, or even better, providing, however, less discomfort for the patient. Thus, a proper planning and diagnosis are essential so that the best treatment can be offered to patients.
\end{abstract}

Key words: Gingiva, Gingivoplasty, Osteotomy, Periodontium, Smiling. 


\section{INTRODUÇÃo}

Aconcepção estéticaé umfator subjetivo, diretamente associado aos padrões sociais e às variações individuais. Genericamente, um sorriso é considerado estético quando apresenta harmonia entre lábios, gengiva, forma, cor e disposição dos elementos dentários. ${ }^{1,2}$

Pesquisas apontam que $10 \%$ da população entre 20 e 30 anos apresentam exposição excessiva de gengiva. ${ }^{3,4}$ Essa condição é mais prevalente em mulheres e tende a regredir gradualmente com a idade, como consequência do aparecimento de flacidez nos lábios superiores e inferiores, que leva a uma diminuição da exposição dos incisivos superiores e um aumento da exposição dos incisivos inferiores. ${ }^{5,6}$

Com a finalidade de melhorar o alinhamento da margem gengival e fornecer uma exposição adequadadosdentes, amodificaçãodocomplexo dentogengival através de procedimentos cirúrgicos é frequentemente indicada nos casos de pacientes que apresentam exposição excessiva de gengiva durante o sorriso. É importante que o profissional esteja atento à causa do problema, que pode ter diferentes etiologias, como: erupção passiva alterada, displasia esquelética ou crescimento excessivo da maxila, uma combinação destes fatores ou ainda o lábio superior curto ou hiperativo ao sorrir. Somente após um correto diagnóstico acerca de qual fator etiológico está presente em cada caso é que um adequado plano de tratamento pode ser proposto. ${ }^{7}$

O objetivo do presente estudo foi descrever um caso clínico de sorriso gengival, mostrando a importância do planejamento e tratamento realizado.

\section{Caso Clínico}

Paciente C.F.S.R., sexo feminino, 27 anos, feoderma, apresentou-se à clínica de Periodontia da PUC-MG com a queixa de dentes curtos (Figura 1). Ao exame clínico foi observada profundidade de sondagem média de $3 \mathrm{~mm}$, e bom controle de biofilme dental. Através da sondagem clínica foi possível identificar a junção amelo cementária (JAC). Realizou-se $O$ exame radiográfico periapical, sugerindo a necessidade de osteoplastia.

Confeccionou-se então um guia cirúrgico para confirmar as informações obtidas através do exame clínico de sondagem transgengival. Confirmou-se que a distância entre o limite amelocementário (LAC) era menor do que 2 $\mathrm{mm}$, caracterizando uma erupção passiva e indicando a técnica cirúrgica da osteoplastia com deslocamento apical do retalho. Assim, para obtenção do modelo, executou-se moldagem com silicone de adição, material indicado para copiar com precisão a anatomia dos dentes e gengiva. O modelo foi confeccionado em gesso especial. Medidas obtidas através de sondagem transgengival foram transferidas para o modelo com lápis cópia, obedecendo-se os princípios estéticos de zênites. Foi confeccionada sobre o modelo uma placa de acrílico para servir como guia cirúrgico. A borda da placa foi evidenciada com cimento de fosfato de zinco com o objetivo de delimitar o limite onde se encontraria o osso. Através desse procedimento de evidenciação do guia com marcador radiopaco, pôde-se sugerir que a imagem tomográfica é confiável para a decisão diagnóstica entre gengivoplastia e erupção passiva. Foi então realizado o exame tomográfico, através da qual confirmou-se a necessidade de osteoplastia (Figuras 2). Foi fechado o diagnóstico de erupção passiva alterada. Assim, o tratamento de escolha foi o aumento de coroa clínica no sextante superior anterior. Após o preparo básico, foi realizada a cirurgia, consistindo de gengivectomia, osteotomia e osteoplastia. Foi realizada a anestesia paraperiostica e local, incisão intrasulcular (Figura 3) e incisão relaxante na mesial dos pré-molares, preservando as papilas. Rebateu-se o retalho (Figura 4), posicionou-se a placa e confirmaram-se as medidas (Figura 
5), e procedeu-se a osteoplastia com cinzel de Oshcheibein e broca cirúrgica cônica multilaminada sob irrigação constante com solução fisiológica. Foi realizada a reposição do retalho, sutura na posição correta e sutura com fio de seda 4 e colocação do cimento cirúrgico (Figura 6). O caso foi acompanhado, o póscirúrgico foi satisfatório, o resultado almejado foi alcançado sem necessidade de cirurgias adicionais (Figura 7). A paciente relatou grande satisfação com o resultado e foi encaminhada para a dentística.

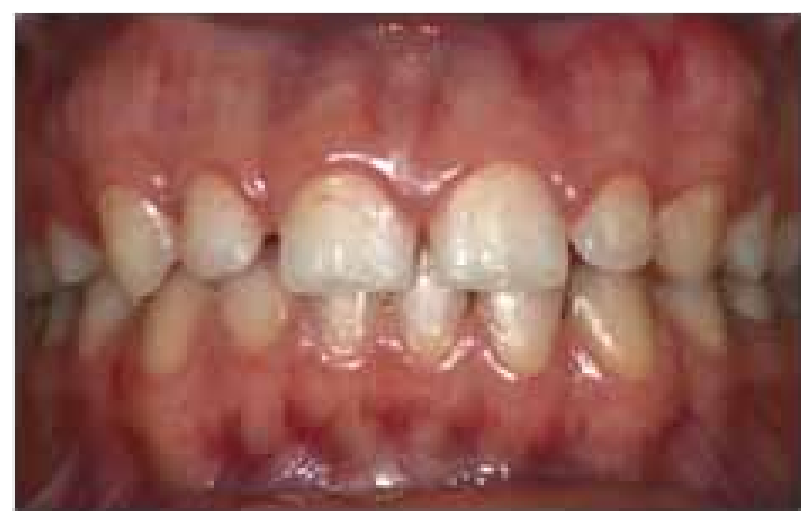

Figura 1. Aspecto inicial.

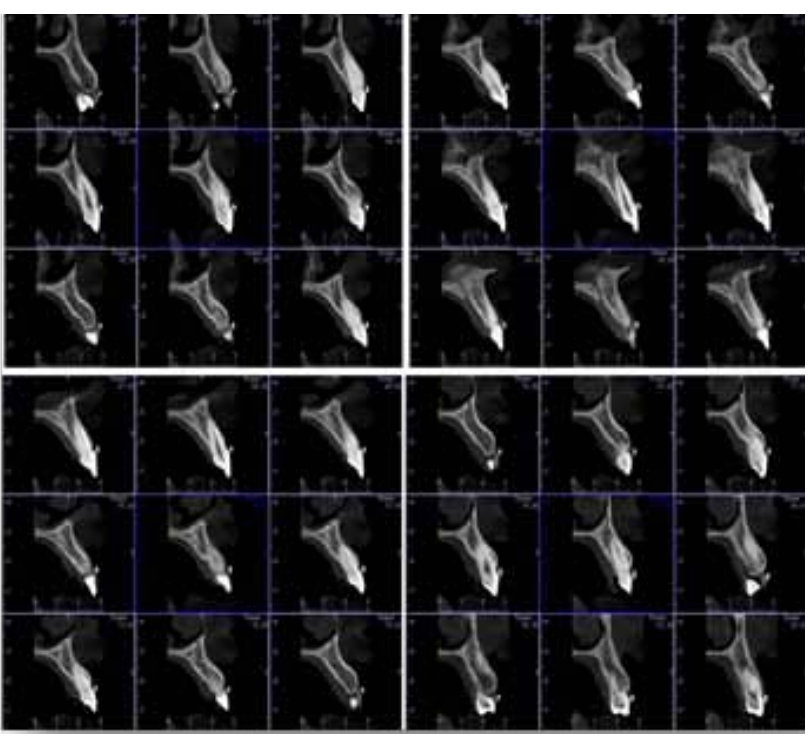

Figura 2. Tomografia transversal de canino a canino.

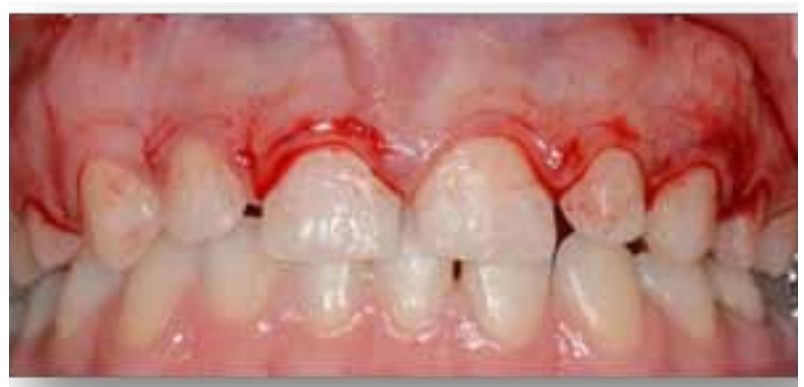

Figura 3. Incisão sulcular

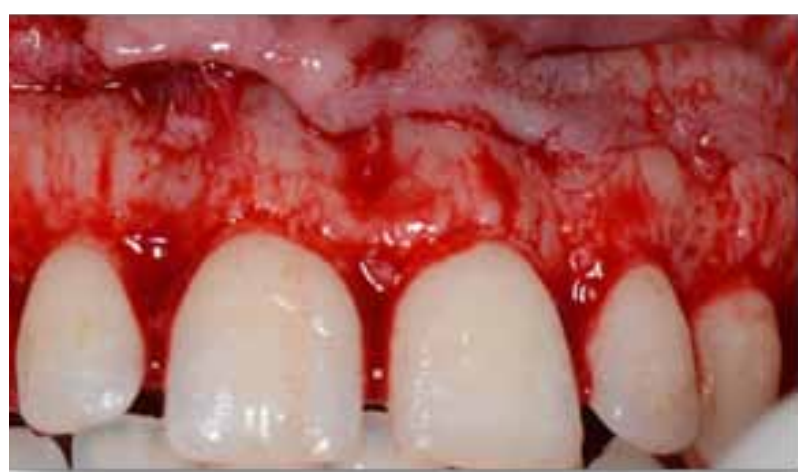

Figura 4. Retalho rebatido

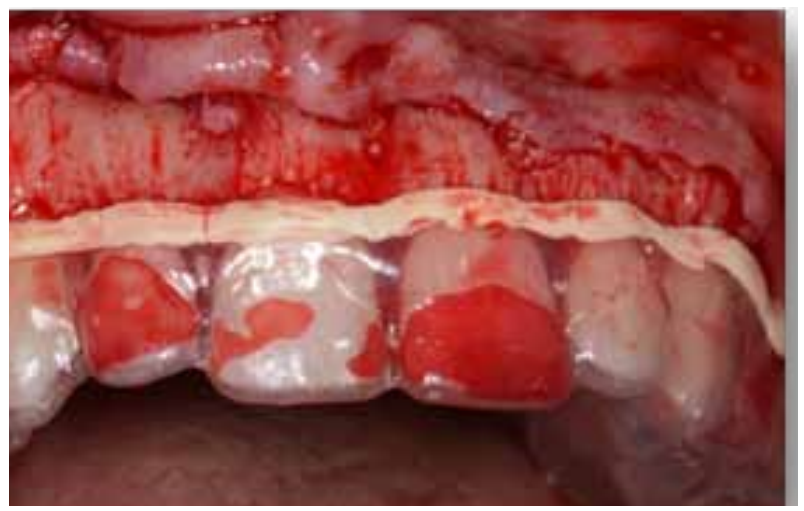

Figura 5. Posicionamento da placa para confirmação das medidas.

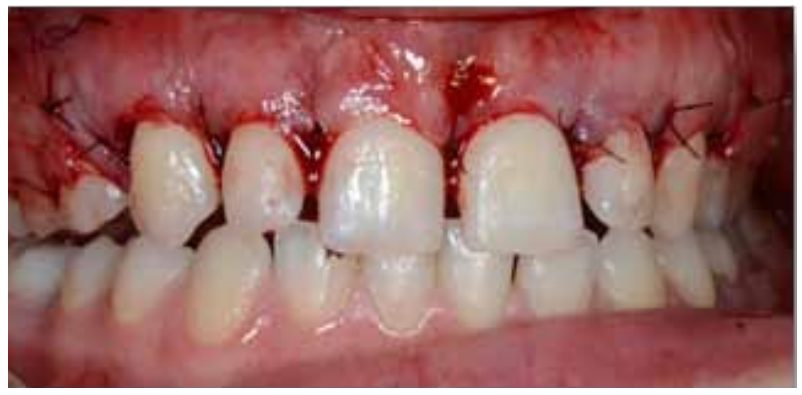

Figura 6. Posicionamento do retalho e sutura. 


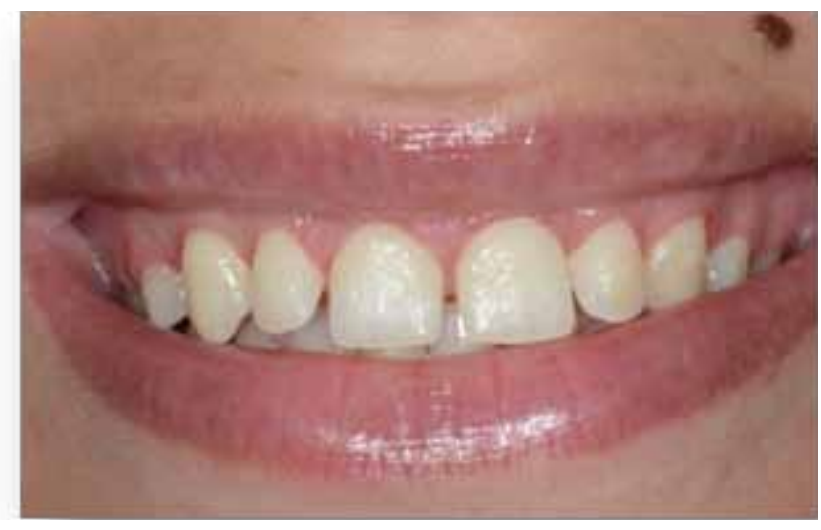

Figura 7. Aspecto final.

\section{Dıscussão}

Os padrões atuais de beleza estão ganhando maior importância e cada vez mais a estética é exigida pelos pacientes nos consultórios odontológicos. A exposição de até $3 \mathrm{~mm}$ de gengiva além dos limites cervicais do dente é aceitável sob o ponto de vista estético. Um sorriso é tido como gengival se mais de $3 \mathrm{~mm}$ de gengiva é visível durante um sorriso moderado. O sorriso forçado permite confirmar essa impressão. Uma quantidade excessiva de tecido mole não é antiestética em si, dependendo sobremaneira da forma cujo excesso está disposto em relação aos dentes e lábios e, essencialmente, da autopercepção do paciente. ${ }^{8}$

Antes do exame intrabucal, é imprescindível a realização de uma anamnese do paciente, em um contexto tão subjetivo quanto o estético. Para isso, é valido questionar o que o paciente acha da estética de sua gengiva e o que espera do tratamento. ${ }^{9}$ Os tecidos gengivais devem ser avaliados por sua dimensão, seu estado de saúde, forma, contorno e cor. Os dentes também devem ser examinados, avaliando o comprimento da coroa clínica (da borda incisal à gengiva marginal) e da coroa anatômica (da borda incisal até a JAC), sendo esta aferível pela sonda periodontal no sulco, salvo as exceções onde esta está abaixo da crista óssea. ${ }^{8}$
Todo caso de sorriso gengival pode ser corrigido, podendo integrarvárias especialidades da Odontologia, uma vez que a causa pode ser bastante variada. ${ }^{10} \mathrm{O}$ crescimento vertical da maxila, que pode ser diagnosticado através de radiografias cefalométricas pela discrepância do osso maxilar, pode ser corrigido através de cirurgia ortognática. Outra possível causa é a hiperfunção dos músculos elevadores do lábio ou lábios curtos, que podem ser corrigidos apenas com aplicação de toxina botulínica. $\mathrm{E}$ outra etiologia bastante relatada é a erupção passiva alterada dos dentes, onde o periodonto não migra satisfatoriamente em sentido apical, recobrindo assim a junção amelocementária, deixando uma coroa clínica pequena e o sorriso semelhante ao de uma criança. Além dessas causas a literatura se refere, em menor freqüência, à extrusão dentoalveolar e a combinação de vários fatores. ${ }^{11}$

A erupção passiva alterada foi classificada tendo como parâmetros a junção mucogengival e a crista óssea. Pode-se nestes casos lançar mão de um método auxiliar para o ato cirúrgico: o planejamento reverso, que consiste na confecção de um guia cirúrgico com diversas funções, tais como: definir plano e arquitetura gengival para as incisões iniciais; estabelecer referência para osteoplastia e a posição do zênite; e simular o resultado esperado. Assim, podemos avaliar o impacto do tratamento proposto e, além disso, o paciente pode interagir e avaliar os resultados esperados. ${ }^{12,13}$

O tratamento ortodôntico ou restaurador pode ser realizado em conjunto como forma de reforçar a estética. Quando não há ampla zona de gengiva inserida, a gengivectomia se encontra contra-indicada, sendo o retalho posicionado apicalmente o tratamento mais indicado. E para o caso onde o sorriso gengival é causado por um crescimento ósseo do osso maxilar, o procedimento de aumento de coroa clinica se encontra limitado, sendo mais indicado para esse caso à correção ortognática. 


\section{Conclusão}

O sorriso gengival compromete a estética do sorriso. Como possui etiologias diversas, é de extrema importância que o cirurgiãodentista realize um exame clínico apurado e, quando necessário, recorra a métodos diagnósticos auxiliares, como os exames radiográficos e as tomografias. Apesar de existirem diversas terapias e tratamentos já consagrados, novas alternativas menos invasivas têm sido encontradas com objetivo de se obter resultados semelhantes aos apresentados pelos tratamentos tradicionais, ou ainda melhores, promovendo, no entanto, um menor desconforto para o paciente. Assim, um planejamento e diagnóstico adequados são essenciais para que o melhor tratamento possa ser oferecido aos pacientes.

\section{REFERÊNCIAS}

1. GOMES, J. C. Odontologia estética restaurações adesivas indiretas. $1^{\circ}$ edição, São Paulo: Artes Médicas, 1996. 213p.

2. MESTRENER, S. R., KOMATSU, J. Recuperação da linha do sorriso utilizando procedimentos cirúrgico e restaurador. J bras dent estét.2010; 1(3).

3. TJAN, A. H., MILLER, G. D. The JGP: Some esthetic factors in a smile. J Prosthet Dent 1984; 51(1): 24-28.

4. PECK, S., PECK, L., KATAJA, M. The gingival smile line. Angle Orthod 1992; 62(2): 91-100.

5. VIG, R. G.; BRUNDO, G. C. The kinetics of anterior tooth display. J Prosthet Dent 1978; 39: 502-4.

6. ZARB, G. A. et al. Prosthodontic Treatment for Edentulous Patients, ed 10. St Louis: Mosby, 1990

7. DE MACEDO, A. C. et al. O sorriso gengival tratamento baseado na etiologia - uma revisão de literatura. Braz J Periodontol, 2012; 22(4): 36-44.
8. BORGHETTI, A., MONNET-CORTI, V. Contribuição da cirurgia plástica periodontal à dentística restauradora em pilares naturais. In: BORGHETTI, A; MONNET-CORTI, V. Cirurgia plástica periodontal. Artmed, 2002.

9. CHEN, L., SCHARER, P. De l'examen au diagnostic em medicine dentaire esthetétique. Real Clin, 1998; 9: 283-91, 1998.

10. FOSQUIERA, E. C., BREMM, L. L., MIURA, C. S. Correção do sorriso gengival associado ao clareamento dental - relato de caso clínico. XVIII Encontro do GBPD. Acesso: 10/05/2014. Disponível em www.uepg.br/encontrogbpd.

11. SILVA, R. C., CARVALHO, P. F., JOLY, J. C. Planejamento estético em periodontia. In: $25^{\circ}$ Congresso Internacional de Odontologia de São Paulo (25 ClOSP); 2007; São Paulo. São Paulo: APCD, 2007

12. GUSMÃO, E. S. et al. Periodontal plastic surgery for smile correction. Odontol clín cient,; 2006; 5(4): 345-8.

13. SUZUKI, P. H. et al. Enhancing the Gingival Smile: Case report. Rev Inpeo de Odontol, 2008; 2(2):1-56.

14. MAZZUCO, R. et al. Gummy smile and botulinum toxin; A new approach based on the gingival esposure area. J Am Acad Dermatol 2012; 63(6):1042-51.

*Endereço para Correspondência:

Sâmila Gonçalves Barra

Rua João de Deus Tavares n45, Barreiro de Cima

Belo Horizonte, MG, Brasil.

Telefone: +55 31 8421-6607

samilagbarra@gmail.com

Submetido em: 26-5-2015

Aceito em: 8-6-2015 